\title{
Venous thromboembolism in non-small cell lung cancer patients who underwent surgery after induction therapy
}

\author{
Yasunori Kaminuma ${ }^{1} \cdot$ Masayuki Tanahashi $^{1} \cdot$ Eriko Suzuki $^{1} \cdot$ Naoko Yoshii $^{1} \cdot$ Hiroshi Niwa $^{1}$
}

Received: 29 January 2020 / Accepted: 31 March 2020 / Published online: 9 April 2020

(c) The Author(s) 2020

\begin{abstract}
Objectives Lung cancer patients have been reported to have a high incidence of venous thromboembolism (VTE) and a high recurrence rate of VTE. However, there are no detailed reports of VTE in lung cancer patients who underwent surgery after induction therapy. We examined the incidence and clinical features of VTE in these patients.

Methods We retrospectively evaluated 89 patients with non-small cell lung cancer who underwent surgery after induction therapy at our department between April 2009 and March 2018. The incidence of VTE, clinical features, and long-term prognosis were retrospectively examined.

Results Among the 89 patients, 4 (4.5\%) developed VTE, and there was no significant difference in the background characteristics between patients with and without VTE. All four patients developed VTE during preoperative treatment. In the patients with VTE, anticoagulant therapy with oral anticoagulants was administered after heparinization, and the median duration of anticoagulant therapy was 18.7 months. There were no cases of symptomatic VTE recurrence after surgery, regardless of lung cancer recurrence. Although the overall survival (OS) showed no significant difference between patients with and without VTE, the disease-free survival was significantly shorter in patients with VTE than in those without it (median 6.3 vs. 71.6 months, $p<0.01$ ).

Conclusions In induction cases, the incidence of VTE was $4.5 \%$, and it can at least be stated that no symptomatic VTE developed or recurred after surgery. Patients with VTE in induction therapy had short progression-free survival and required careful follow-up after surgery.
\end{abstract}

Keywords Non-small cell lung cancer · Venous thromboembolism · Pulmonary embolism · Deep vein thrombosis . Induction therapy

Postscript: The outline of this study was presented at the 72nd Annual Scientific Meeting of The Japanese Association for Thoracic Surgery in Kyoto, Japan.

Yasunori Kaminuma

kouhan.work@gmail.com

Masayuki Tanahashi

m.tanahashi@sis.seirei.or.jp

Eriko Suzuki

e-suzuki@sis.seirei.or.jp

Naoko Yoshii

n-yoshii@sis.seirei.or.jp

Hiroshi Niwa

niwah@sis.seirei.or.jp

1 Division of Thoracic Surgery, Respiratory Disease Center, Seirei Mikatahara General Hospital, 3453, Mikatahara-Cho, Kita-Ku, Hamamatsu, Shizuoka 433-8558, Japan

\section{Introduction}

Venous thromboembolism (VTE) consists of pulmonary thromboembolism (PE) and deep vein thrombosis (DVT). VTE occurs in 4-20\% of cancer patients, and lung cancer is reported to carry a high risk of VTE complications among solid tumors [1-3]. Cancer patients also have a high VTE recurrence rate [4]. Thrombus formation is promoted as follows: coagulation factors are activated by tissue factors and procoagulants secreted from cancer cells, and vascular endothelial cells are damaged by mucin-like substances and cytokines derived from cancer cells [1]. Patients with unresectable/recurrent cancer are at a high risk of VTE recurrence, due to the persistent hypercoagulable state caused by cancer. However, if a cancer-free status can be obtained by surgery after induction treatment for advanced lung cancer, the internal environment surrounding VTE may differ from 
that in patients with unresectable/recurrent cancer. In this study, we clarified the incidence rate, high incidence period, and recurrence rate of VTE in patients who received induction therapy, and examined the long-term prognosis of these patients.

\section{Patients and methods}

We included 89 patients with non-small cell lung cancer (NSCLC) who underwent surgery after induction therapy in our department from April 2009 to March 2019. The incidence of VTE, clinical background characteristics, and the recurrence rate were examined retrospectively. We divided the treatment phase into induction, perioperative, and follow-up periods, and examined which periods were more likely to develop VTE (Fig. 1). The inclusion criteria for induction therapy were patients with mediastinal lymph node metastasis (N2 or 3) and/or direct invasion of nearby organs (T3 or 4), up to 70-75 years old, and an Eastern Cooperative Oncology Group performance status (ECOG-PS) of 0. The neoadjuvant chemotherapy regimen was platinum-based doublet chemotherapy, and radiation therapy with 40-50 Gy was performed whenever possible. Before the start of treatment, contrast-enhanced computed tomography (CT), positron emission tomography (PET)-CT, and brain magnetic resonance imaging (MRI) were performed, and all patients were histologically diagnosed with NSCLC. In patients for whom lymph node metastasis was suspected, a histological evaluation was performed with endobronchial ultrasoundguided transbronchial needle aspiration (EBUS-TBNA) or mediastinoscopy whenever possible. The 8th edition of the TNM classification was used to determine the lung cancer staging, and the therapeutic effect was judged according to the Response Evaluation Criteria in Solid Tumors version 1.1 (RESIST v.1.1).
Contrast-enhanced CT was performed in all cases when clinical symptoms suggestive of VTE appear during treatment and when determining the effect of preoperative therapy.

Regardless of the presence or absence of symptoms, anticoagulation with unfractionated heparin (UFH) was immediately performed when VTE was detected on contrast-enhanced CT. After that, the medication was changed to an oral anticoagulant and continued to be taken until the patient's condition stabilized. Exclusion criteria were patients in whom the absence of VTE could not be confirmed before the start of induction therapy, those who started preoperative treatment but for whom surgery was deemed difficult, and those in whom contrast CT was deemed difficult. We also excluded patients with non-controllable infections, diabetes, hypertension, and severe pre-existing conditions, including double cancer in need of treatment.

This study was approved by the ethics committee of our institution (authorization number 19-20). The requirement of informed consent from each patient was waived due to the retrospective study of the data.

\section{Statistical analyses}

Regarding the statistical methods, Fisher's exact test and the Mann-Whitney $U$ test were used for background comparisons. The survival curve was calculated by the Kaplan-Meier method. The log-rank test was used for the significance test, and significant differences were determined at $p<0.05$. All statistical analyses were performed with $\mathrm{R}$ (The R Foundation for Statistical Computing, Vienna, Austria) and EZR (Saitama Medical Center, Jichi Medical University, Saitama, Japan), which is a modified version of $\mathrm{R}$ commander designed to add statistical functions frequently used in biostatistics [5].

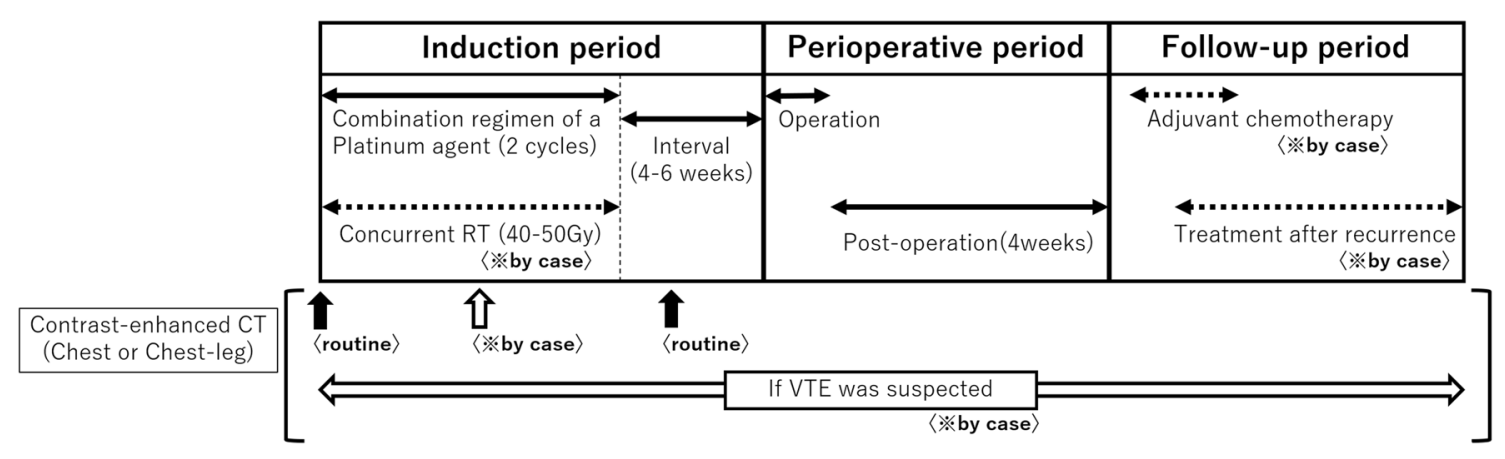

Fig. 1 Diagram for the treatment and contrast enhanced-CT (chest or chest-leg) follow-up. The treatment phase was divided into induction, perioperative, and follow-up periods. We examined in which periods VTE was more likely to develop. Routine contrast enhanced-CT follow-up was performed before induction therapy and when assessing the therapeutic effects of induction therapy. In other cases, we performed contrast-enhanced CT when assessing the therapeutic effects at the midpoint of induction therapy and when symptomatic VTE was clinically suspected 


\section{Results}

\section{Patient characteristics}

Of the 89 patients undergoing induction therapy, 4 (4.5\%) developed VTE. There was no apparent significant difference in the background of patients with and without VTE (Table 1). The smoking history, stage, histological type and pathological therapeutic response varied among patients with VTE. However, the one thing that they all had in common was the use of cisplatin-based chemotherapy and radiation combination therapy. (Table 2).

All patients with VTE had PE and DVT, but they were asymptomatic. In all cases, VTE was diagnosed accidentally by contrast-enhanced CT performed during and at the end of induction therapy. The median period of VTE onset from the start of induction therapy was 47 days (24-56 days), and all patients developed VTE during the induction period. In patients with VTE, after the administration of UFH, oral anticoagulants (Warfarin/Edoxaban $=2 / 2$ cases) were continuously administered for a median of 18.7 months (6.2-24.9 months). The anticoagulant was discontinued before the operation and resumed early after the operation. There were no adverse events associated with anticoagulant therapy, including bleeding, and there was no recurrence of VTE after discontinuation.

\section{The prognosis}

There was no significant difference in the 5-year overall survival (OS) when comparing the prognosis of the patients with and without VTE (log-rank test, $p=0.53$ ). The 5-year OS rate of the patients with VTE was not reached, and the median OS was 35.9 months (95\% confidence interval $[\mathrm{Cl}]$ 26.0-N/A months). The 5-year OS rate of the patients without VTE was $60.3 \%$, and the median OS was 83.3 months (95\% Cl 52.9-N/A months) (Fig. 2).

There was a significant difference in the 5-year disease-free survival (DFS) when comparing the prognosis of the patients with and without VTE, with the patients with VTE showing a significantly worse prognosis (logrank test, $p<0.01)$. The 5-year DFS rate of the patient with VTE was $0 \%$, and the median DFS was 6.3 months (95\% Cl 2.9-N/A months). The 5-year DFS rate of the patients without VTE was $51.9 \%$, and the median DFS was 71.6 months (95\% Cl 23.9-N/A months) (Fig. 3).

Prognostic factors in induction therapy cases were examined by a univariate analysis, and there were no significant prognostic factors for the OS. In DFS, the presence of VTE and a poor pathological therapeutic response (Ef.0/1) were significant prognostic factors $(p=0.02$ and $<0.01)$ (Table 3 ).

\section{Discussion}

The general incidence of VTE is reported to be 4-20\% in cancer patients [1-3]. In the present study, the frequency was equivalent to $4.5 \%$ in lung cancer patients who underwent surgery after induction therapy. In a trial related to induction therapy for lung cancer, VTE was reported as an adverse event in $4 \%$ of cases. Given the above, the incidence of VTE in patients who undergo surgery after induction therapy is expected to be around 4-5\% [6]. Chemotherapy with cisplatin has been reported to carry a significantly higher incidence of VTE than other platinum-based chemotherapy regimens, and all VTE cases were in the cisplatin combination group in the present study [7]. In addition, the recurrence rate of PE was reportedly significantly higher and the risk of cerebral hemorrhaging higher in cases in which chemoradiation therapy was performed than in others. However, $\mathrm{PE}$ recurrence and bleeding events were not observed in the present study [8]. In the studies of non-induction therapy, a dissertation reported the postoperative rate of VTE changes depending on the extent of operation (lobectomy or bilobectomy vs. pneumonectomy) and surgical approach (videoassisted thoracic surgery vs. thoracotomy) [9]. However, another study reported that the incidence of VTE did not differ according to the surgical approach (minimally invasive surgery vs. open surgery) or the extent of operation (lobectomy, wedge resection and pneumonectomy) [10]. There was no fixed consensus on the relationship between the incidence of postoperative VTE and the surgical procedures. In induction therapy for lung cancer, the causal relationship between the surgical procedures and the incidence of VTE remains unclear, because there were no postoperative cases of VTE in our study and no reports have examined the incidence of VTE.

All VTE cases in our study were asymptomatic. Anticoagulant therapy is required, regardless of symptoms, as there have been reports that anticoagulant therapy for asymptomatic DVT significantly reduces the appearance and recurrence of symptomatic VTE in patients with cancer [11]. European and American guidelines recommend low-molecular-weight heparin (LMWH) for VTE patients with cancer, but LMWH is not available in Japan. Therefore, after treatment with UFH, anticoagulant therapy with oral coagulants (Warfarin) is often continued. However, direct oral anticoagulants (DOACs) have recently been reported to prevent recurrence, and DOACs are commonly used because of their low risk of bleeding and drug interaction [12]. There is no fixed view concerning the duration of treatment, but in recent years, anticoagulant therapy for half a year or more has been recommended. The present study included cases for which the guidelines and consensus were unclear. These patients were therefore given warfarin 
Table 1 Characteristics of patients with and without VTE undergoing induction therapy followed by surgery

\begin{tabular}{|c|c|c|c|c|}
\hline & $\begin{array}{l}\text { Total } \\
n=89\end{array}$ & $\begin{array}{l}\text { Patients with VTE } \\
n=4\end{array}$ & $\begin{array}{l}\text { Patients without VTE } \\
n=85\end{array}$ & $p$ value \\
\hline Sex & & & & 0.502 \\
\hline Male & $75(84.3 \%)$ & $3(75.0 \%)$ & $72(84.7 \%)$ & \\
\hline Female & $14(15.7 \%)$ & $1(25.0 \%)$ & $13(15.3 \%)$ & \\
\hline Age & & & & 0.186 \\
\hline Median (range) & $62.0(36-73)$ & $60.5(52-71)$ & $59.9(36-73)$ & \\
\hline ECOG PS & & & & 1 \\
\hline 0 & $89(100 \%)$ & $4(100 \%)$ & $85(100 \%)$ & \\
\hline Smoking status & & & & 0.319 \\
\hline Never smoker & $8(9.0 \%)$ & $1(25.0 \%)$ & $7(8.2 \%)$ & \\
\hline Former or current & $81(91.0 \%)$ & $3(75.0 \%)$ & $78(91.8 \%)$ & \\
\hline Smoking index (pack years) & & & & 0.324 \\
\hline Median (range) & $37.4(0-106)$ & $48.3(0-106)$ & $36.9(0-82)$ & \\
\hline CT total tumor size & & & & 0.804 \\
\hline Median (range) & $5.1(1.1-30)$ & $4.6(1.7-10.0)$ & $5.1(1.1-30)$ & \\
\hline PET-CT SUVmax & & & & 0.376 \\
\hline Median (range) & $12.2(2.8-25.0)$ & $14.4(2.8-22.0)$ & $12.1(3.3-25.0)$ & \\
\hline Histology & & & & 0.186 \\
\hline Squamous & $30(33.7 \%)$ & $2(50.0 \%)$ & $28(32.9 \%)$ & \\
\hline Adenocarcinoma & $49(55.1 \%)$ & $1(25.0 \%)$ & $48(56.5 \%)$ & \\
\hline Adenosquamous & $3(3.4 \%)$ & $1(25.0 \%)$ & $2(2.4 \%)$ & \\
\hline Pleomorphic carcinoma & $3(3.4 \%)$ & $0(0.0 \%)$ & $3(3.5 \%)$ & \\
\hline NSCLC & $4(4.5 \%)$ & $0(0.0 \%)$ & $4(4.7 \%)$ & \\
\hline Clinical $\mathrm{T}$ factor & & & & 0.09 \\
\hline $\mathrm{T} 1$ & $15(16.9 \%)$ & $2(50.0 \%)$ & $13(15.3 \%)$ & \\
\hline $\mathrm{T} 2$ & $15(16.9 \%)$ & $0(0.0 \%)$ & $15(17.6 \%)$ & \\
\hline $\mathrm{T} 3$ & $29(32.6 \%)$ & $2(50.0 \%)$ & $27(31.8 \%)$ & \\
\hline $\mathrm{T} 4$ & $30(33.7 \%)$ & $0(0.0 \%)$ & $30(35.3 \%)$ & \\
\hline Cinical $\mathrm{N}$ factor & & & & 1 \\
\hline No & $30(33.7 \%)$ & $2(50.0 \%)$ & $28(32.9 \%)$ & \\
\hline N1 & $11(12.4 \%)$ & $0(0.0 \%)$ & $11(12.9 \%)$ & \\
\hline $\mathrm{N} 2$ & $42(47.2 \%)$ & $2(50.0 \%)$ & $40(47.1 \%)$ & \\
\hline N3 & $6(6.7 \%)$ & $0(0.0 \%)$ & $6(7.1 \%)$ & \\
\hline Clinical stage & & & & 0.543 \\
\hline IIB & $22(24.7 \%)$ & $2(50.0 \%)$ & $20(23.5 \%)$ & \\
\hline IIIA & $47(52.8 \%)$ & $2(50.0 \%)$ & $45(52.9 \%)$ & \\
\hline IIIB & $19(21.4 \%)$ & $0(0.0 \%)$ & $19(22.4 \%)$ & \\
\hline IIIC & $1(1.1 \%)$ & $0(0.0 \%)$ & $1(1.2 \%)$ & \\
\hline Regimen of neoadjuvant therapy & & & & 1 \\
\hline $\mathrm{CDDP}+\mathrm{DTX}$ & $40(44.9 \%)$ & $2(50.0 \%)$ & $38(44.7 \%)$ & \\
\hline $\mathrm{CDDP}+\mathrm{VNR}$ & $36(40.5 \%)$ & $2(50.0 \%)$ & $34(40.0 \%)$ & \\
\hline $\mathrm{CBDCA}+\mathrm{PTX}$ & $11(12.4 \%)$ & $0(0.0 \%)$ & $11(12.9 \%)$ & \\
\hline CBDCA + DTX & $2(2.3 \%)$ & $0(0.0 \%)$ & $2(2.4 \%)$ & \\
\hline Radiation dose of neoadjuvant therapy & & & & 0.426 \\
\hline 40 Gy & $32(36.0 \%)$ & $3(75.0 \%)$ & $29(34.1 \%)$ & \\
\hline $50 \mathrm{~Gy}$ & $47(52.8 \%)$ & $1(25.0 \%)$ & $46(54.1 \%)$ & \\
\hline None & $10(11.2 \%)$ & $0(0.0 \%)$ & $10(11.8 \%)$ & \\
\hline RECIST tumor response & & & & 1 \\
\hline Complete response & $1(1.1 \%)$ & $0(0.0 \%)$ & $1(1.2 \%)$ & \\
\hline Partial response & $46(51.7 \%)$ & $2(50.0 \%)$ & $44(51.8 \%)$ & \\
\hline
\end{tabular}


Table 1 (continued)

\begin{tabular}{llll}
\hline & $\begin{array}{l}\text { Total } \\
n=89\end{array}$ & $\begin{array}{l}\text { Patients with VTE } \\
n=4\end{array}$ & $\begin{array}{l}\text { Patients without VTE } \\
n=85\end{array}$ \\
\hline Stable disease & $41(46.1 \%)$ & $2(50.0 \%)$ & $39(45.9 \%)$ \\
Progressive disease & $1(1.1 \%)$ & $0(0.0 \%)$ & $1(1.2 \%)$ \\
Pathological therapeutic response & & & $1(1.2 \%)$ \\
Ineffective (Ef.0) & $1(1.1 \%)$ & $0(0.0 \%)$ & $34(40.0 \%)$ \\
Slightly effective (Ef.1) & $35(39.3 \%)$ & $1(25.0 \%)$ & $35(41.2 \%)$ \\
Moderately effective (Ef.2) & $37(41.6 \%)$ & $2(50.0 \%)$ & $15(17.6 \%)$ \\
Markedly effective (Ef.3) & $16(18.0 \%)$ & $1(25.0 \%)$ & $76(89.4 \%)$ \\
Complete resection & $80(89.9 \%)$ & $4(100.0 \%)$ & $9(10.6 \%)$ \\
Incomplete resection & $9(10.1 \%)$ & $0(0.0 \%)$ & $36(42.4 \%)$ \\
No recurrent survival & $38(42.7 \%)$ & $2(50.0 \%)$ & $15(17.6 \%)$ \\
Recurrent survival & $15(16.9 \%)$ & $0(0.0 \%)$ & $34(40.0 \%)$ \\
Death & $36(41.5 \%)$ & $2(50.0 \%)$ & 22 \\
Cancer death & 24 & 2 & 1
\end{tabular}

Pathological therapeutic response: Ef 0, no therapeutic effect; Ef 1a, residual viable cancer cells detected in " $\geq 2 / 3$ " of resected tumor; Ef $1 \mathrm{~b}$, residual viable cancer cells detected in " $<2 / 3$ and $\geq 1 / 3$ " of resected tumor; Ef 2 , residual viable cancer cells detected in " $<1 / 3$ " of resected tumor; Ef 3, no residual viable cancer cells [14]

PET-CT positron emission tomography-computed tomography, SUVmax maximum standardized uptake value, NSCLC non-small cell lung cancer, $C D D P$ cisplatin, $D T X$ docetaxel, VNR vinorelbine, $C B D C A$ carboplatin, $P T X$ paclitaxel, VTE venous thromboembolism

after the administration of UFH for about 2 years (cases 1,2). Edoxaban has recently been used as a DOAC, with anticoagulant therapy performed for half a year (cases 3 , 4). Patients with unresectable/relapsed cancer have been reported to have a $20.7 \%$ risk of VTE recurrence, even if anticoagulant therapy is used [4]. In the present study, all patients developed VTE during the induction period, but had no recurrence after surgery or discontinuation of anticoagulation therapy, so anticoagulation therapy beyond the recommended period is considered unnecessary.

The DFS was significantly shorter in patients with VTE than in those without VTE (median 6.3 vs. 71.6 months, $p<0.01$ ), and a univariate analysis revealed that the presence of VTE was a poor prognostic factor for the DFS. The short progression-free survival in patients with VTE might have been due to the interaction between thrombi and circulating microcancer in the blood. Substances such as tumor-derived mucin and cytokines damage the vascular epithelium and promote the formation of microthrombi in blood vessels. The microthrombi can trap cancer cells that circulate in the blood and thus function as a scaffold for metastasis [13]. Metastasis, especially distant metastasis, was considered more likely to occur in patients with VTE than in those without it, because metastasis of circulating microcancer in the blood is likely to occur due to the effect of the microthrombi.

The VTE recurrence rate of cancer-bearing patients is reportedly about $30 \%$, but there were no case of recurrence of symptomatic VTE in this study [4]. This suggests that the disease state in the patients who develop VTE during induction therapy may differ from that in patients with unresectable/recurrent cancer. Once lung cancer is removed via surgery after induction therapy, patients become cancer free, thereby resetting their abnormalities in the coagulation system. Recurrence of VTE is thus expected to be less frequent in induction therapy cases than in unresectable/recurrent cases. However, this is only a hypothesis, and precisely why VTE does not develop after recurrence is unclear at present.

The median recurrence period for VTE cases was 6.3 months, and careful follow-up is necessary from the early postoperative period. VTE patients are reported to have a poor prognosis, but the 5-year OS was similar between patients with and without VTE in this study [3].

This study is limited by its retrospective nature, and the fact that there were only 89 patients and 4 patients with VTE, a small number. Verification in a large, prospective study is therefore necessary. Another major limitation of this study was the inability to make a direct comparison with patients with unresectable/recurrent cancer or patients who had undergone non-surgical treatment, such as radical chemoradiation therapy. In addition, regular enhanced CT and coagulation tests were not performed. Asymptomatic VTE cases were therefore not diagnosed, while contrast-enhanced CT is actively performed for symptomatic VTE. The actual onset rate of total VTE may be higher than that depicted in this study. 

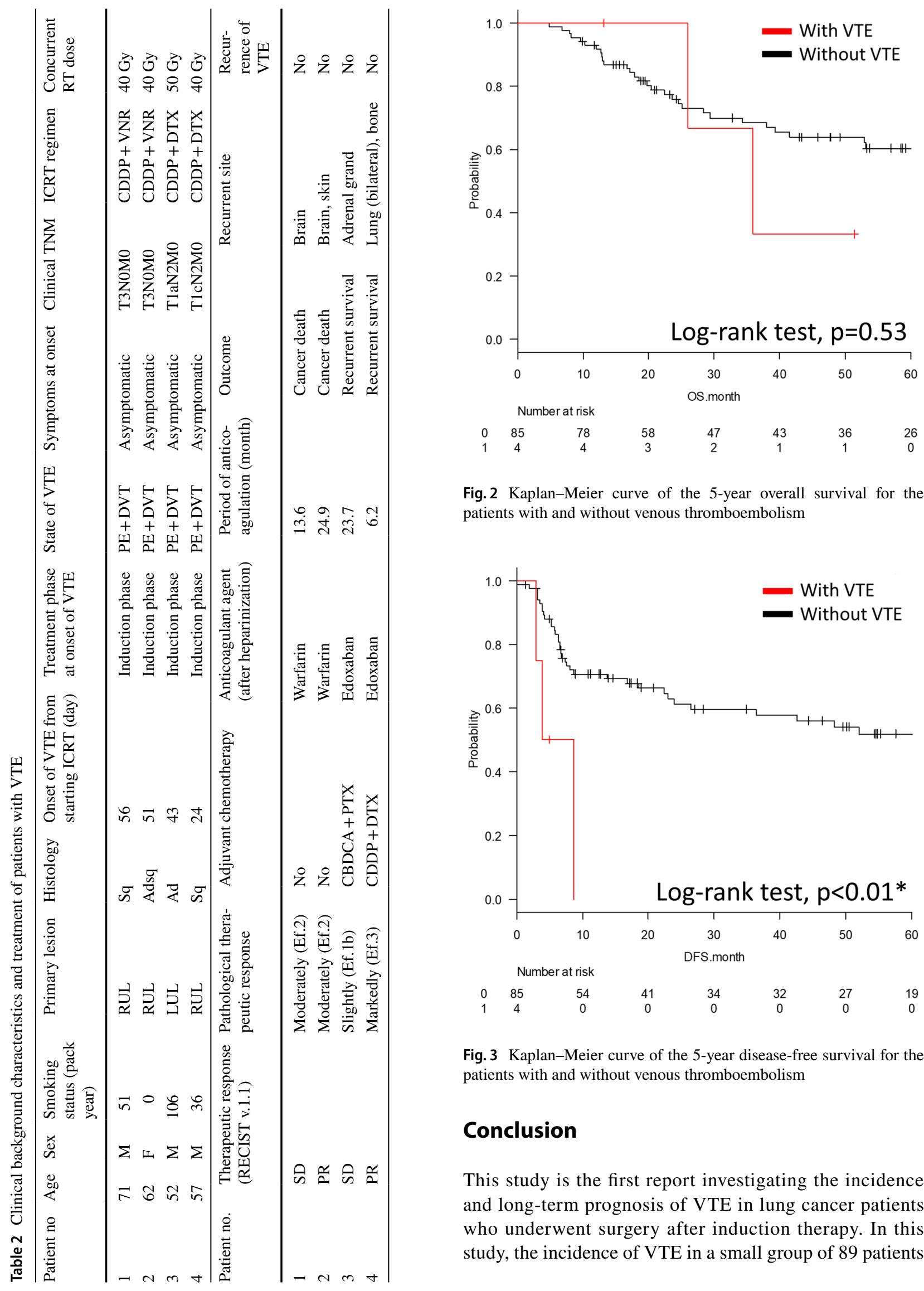

Fig. 2 Kaplan-Meier curve of the 5-year overall survival for the patients with and without venous thromboembolism

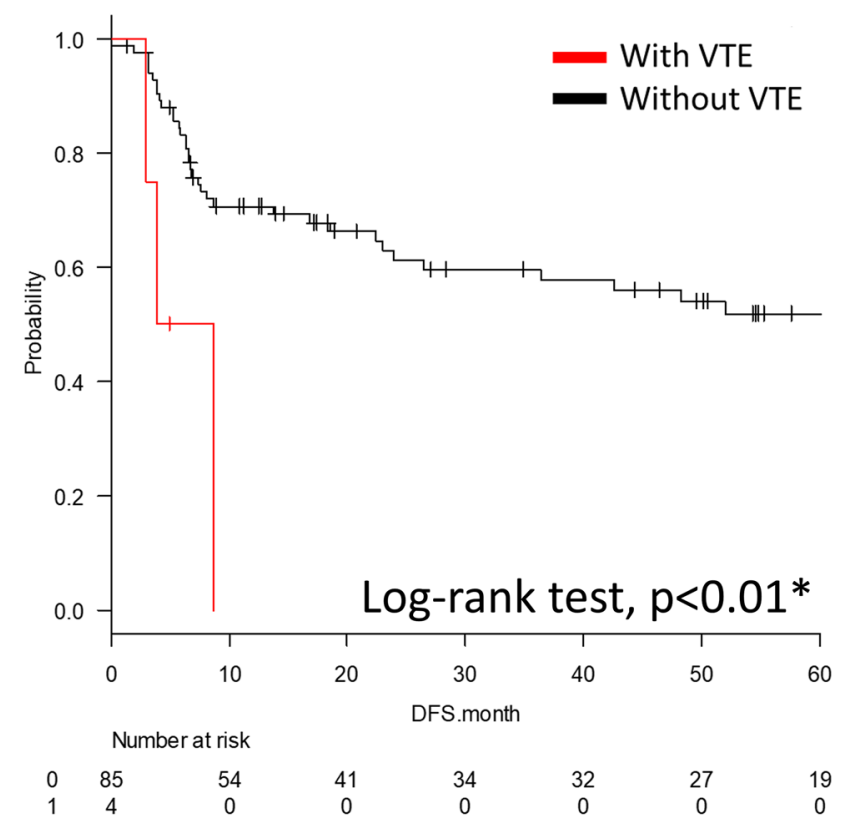

Fig. 3 Kaplan-Meier curve of the 5-year disease-free survival for the patients with and without venous thromboembolism

\section{Conclusion}

This study is the first report investigating the incidence and long-term prognosis of VTE in lung cancer patients who underwent surgery after induction therapy. In this study, the incidence of VTE in a small group of 89 patients 
Table 3 Results of a univariate analysis of the predictive factors for the 5-year overall and disease-free survival

\begin{tabular}{llc}
\hline & $\begin{array}{l}\text { Overall } \\
\text { survival } \\
p\end{array}$ & $\begin{array}{l}\text { Disease- } \\
\text { free } \\
\text { survival } \\
p\end{array}$ \\
\hline Sex & 0.61 & 0.17 \\
Age (over 65 years) & 0.87 & 0.89 \\
Smoking (never- vs. others) & 0.96 & 0.86 \\
Histology (adenocarcinoma vs. others) & 0.05 & 0.90 \\
cStage (II vs. III) & 0.32 & 0.80 \\
RT (0 Gy vs. 40-50 Gy) & 0.75 & 0.77 \\
RECIST (CR/PR vs. SD/PD) & 0.52 & 0.11 \\
Complete resection & 0.10 & 0.09 \\
Pathological therapeutic response (Ef.2/3 or & 0.23 & 0.02 \\
less) & & \\
VTE event & 0.53 & $<0.01$ \\
\hline
\end{tabular}

*Significant difference at a probability level of 0.05

was $4.5 \%$. It can at least be stated that no "symptomatic" VTE developed or recurred after surgery. DFS may be significantly shorter in patients who develop VTE during induction therapy. Careful follow-up is necessary, as cancer recurrence is seen early after surgery in patients with VTE.

Funding No funding was provided.

\section{Compliance with ethical standards}

Conflict of interest The authors have no conflict of interest to declare.

Open Access This article is licensed under a Creative Commons Attribution 4.0 International License, which permits use, sharing, adaptation, distribution and reproduction in any medium or format, as long as you give appropriate credit to the original author(s) and the source, provide a link to the Creative Commons licence, and indicate if changes were made. The images or other third party material in this article are included in the article's Creative Commons licence, unless indicated otherwise in a credit line to the material. If material is not included in the article's Creative Commons licence and your intended use is not permitted by statutory regulation or exceeds the permitted use, you will need to obtain permission directly from the copyright holder. To view a copy of this licence, visit http://creativecommons.org/licenses/by/4.0/.
2. Horsted F, West J, Grainge MJ. Risk of venous thromboembolism in patients with cancer: a systematic review and meta-analysis. PLoS Med. 2012;9(7):e1001275.

3. Ay C, Pabinger I, Cohen AT. Cancer-associated venous thromboembolism; burden, mechanisms, and management. Thromb Haemost. 2017;117(2):219-30.

4. Prandoni P, Lensing AW, Piccioli A, Bernardi E, Simioni P, Girolami B, et al. Recurrent venous thromboembolism and bleeding complications during anticoagulant treatment in patients with cancer and venous thrombosis. Blood. 2002;100(10):3484-8.

5. Kanda Y. Investigation of the freely available easy-to-use software 'EZR' for medical statistics. Bone Marrow Transplant. 2013;48(3):452-8.

6. Kawaguchi K, Yokoi K, Niwa H, Ohde Y, Mori S, Okumura $S$, et al. Trimodality therapy for lung cancer with chest wall invasion: initial results of a phase II study. Ann Thorac Surg. 2014;98(4):1184-91.

7. Zahir MN, Shaikh Q, Shabbir-Moosajee M, Jabbar AA. Incidence of venous thromboembolism in cancer patients treated with cisplatin based chemotherapy—a cohort study. BMC Cancer. 2017;17(1):57.

8. Guy JB, Bertoletti L, Magné N, Rancoule C, Mahé I, Font C, et al. Venous thromboembolism in radiation therapy cancer patients: findings from the RIETE registry. Crit Rev Oncol Hematol. 2017;113:83-9.

9. Thomas DC, Arnold BN, Hoag JR, Salazar MC, Detterbeck FC, Boffa DJ, et al. Timing and risk factors associated with venous thromboembolism after lung cancer resection. Ann Thorac Surg. 2018;105:1469-75.

10. Song C, Shargall Y, Li H, Tian B, Chen S, Miao J, et al. Prevalence of venous thromboembolism after lung surgery in China: a single-centre, prospective cohort study involving patients undergoing lung resections without perioperative venous thromboembolism prophylaxis. Eur J Cardiothorac Surg. 2019;55:455-60.

11. National Clinical Guideline Centre (UK) (2012). Venous thromboembolic diseases: the management of venous thromboembolic diseases and the role of thrombophilia testing. https://www.ncbi. nlm.nih.gov/books/NBK132796/. Accessed 6 Mar 2020.

12. Raskob GE, van Es N, Verhamme P, Carrier M, Di Nisio M, Garcia D, et al. Edoxaban for the treatment of cancer-associated venous thromboembolism. N Engl J Med. 2018;378(7):615-24.

13. Demers M, Wagner DD. Neutrophil extracellular traps: a new link to cancer-associated thrombosis and potential implications for tumor progression. Oncoimmunology. 2013;2(2):e22946.

14. Tanaka F, Yokomise H, Soejima T, Uramoto H, Yamanaka T, Nakagawa K, et al. Induction chemoradiotherapy (50 Gy), followed by resection, for stage IIIA-N2 non-small cell lung cancer. Ann Thorac Surg. 2018;106(4):1018-24.

Publisher's Note Springer Nature remains neutral with regard to jurisdictional claims in published maps and institutional affiliations.

\section{References}

1. Mukai M, Oka T. Mechanism and management of cancer-associated thrombosis. J Cardiol. 2018;72(2):89-93. 